\title{
Asymptomatic Carotid Artery Stenosis Treated with Medical Therapy Alone: Temporal Trends and Implications for Risk Assessment and the Design of Future Studies
}

\author{
Nira Hadar ${ }^{a, b}$ Gowri Raman ${ }^{c}$ Denish Moorthy ${ }^{c}$ Thomas F. O'Donnell ${ }^{d}$ \\ David E. Thalere Edward Feldmann ${ }^{e}$ Joseph Lau ${ }^{a, b}$ Georgios D. Kitsios ${ }^{f}$ \\ Issa J. Dahabreh ${ }^{a, b}$ \\ ${ }^{a}$ Center for Evidence-Based Medicine, and Department of ${ }^{b}$ Health Services, Policy and Practice, School of Public Health, \\ Brown University, Providence, R.I., 'Center for Clinical Evidence Synthesis, Institute for Clinical Research and Health \\ Policy Studies, and Departments of d Vascular Surgery and e Neurology, Tufts Medical Center, Boston, Mass., and \\ fDivision of Pulmonary, Allergy and Critical Care Medicine, University of Pittsburgh Medical Center, Pittsburgh, Pa., USA
}

\section{Key Words}

Asymptomatic carotid stenosis - Medical therapy .

Meta-analysis · Meta-regression

\begin{abstract}
Background: The rate of adverse clinical outcomes among patients with asymptomatic carotid stenosis receiving medical therapy alone can be used to guide clinical decisionmaking and to inform future research. We aimed to investigate temporal changes in the incidence rate of clinical outcomes among patients with asymptomatic carotid stenosis receiving medical therapy alone and to explore the implications of these changes for the design of future comparative studies. Summary: We searched MEDLINE, the Cochrane Central Register of Controlled Trials, US Food and Drug Administration documents, and reference lists of included studies (last search: December 31, 2012). We selected prospective cohort studies of medical therapy for asymptomatic carotid artery stenosis and we extracted information on
\end{abstract}

study characteristics, risk of bias, and outcomes. We performed meta-analyses to estimate summary incidence rates, meta-regressions to assess trends over time, and simulations to explore sample size requirements for the design of future studies comparing new treatments against medical therapy. The main outcomes of interest were ipsilateral stroke, any stroke, cardiovascular death, death, and myocardial infarction. We identified 41 studies of medical therapy for patients with asymptomatic carotid stenosis (last recruitment year: 1978-2009). The summary incidence rate of ipsilateral carotid territory stroke ( 25 studies) was 1.7 per 100 person-years. This incidence rate was significantly lower in recent studies (last recruitment year from 2000 onwards) as compared to studies that ended recruitment earlier (1.0 vs. 2.3 events per 100 person-years; $p<0.001)$. The incidence rates of any territory stroke (17 studies), cardiovascular death (6 studies), death (13 studies), and myocardial infarction ( 5 studies) were 2.7, 4.1, 4.6, and 1.8 per 100 person-years, respectively. Simulations showed that future studies would need to enroll large numbers of patients with a relatively high incidence rate un-

\section{KARGER}

E-Mail karger@karger.com www.karger.com/ced
(C) 2014 S. Karger AG, Basel

1015-9770/14/0383-0163\$39.50/0
Nira Hadar

Center for Evidence-Based Medicine

School of Public Health, Brown University

Providence, RI 02912 (USA)

E-Mail nira_hadar@brown.edu 
der medical therapy, and evaluate interventions with large effect sizes, to have adequate power to reliably detect treatment effects. Key Messages: Improved prognosis under medical therapy alone has narrowed the potential range of risk reduction attainable with new treatments for asymptomatic carotid stenosis. Future comparative studies will need to enroll large numbers of patients to assess treatment effectiveness.

(c) 2014 S. Karger AG, Basel

\section{Introduction}

Carotid artery stenosis is an important cause of ischemic stroke and is increasingly prevalent in the aging US population. Because carotid artery atherosclerosis can progress silently during an asymptomatic phase, its first clinical manifestation can be a debilitating or fatal stroke [1]. Treatment options for the management of carotid stenosis include surgical endarterectomy or percutaneous carotid angioplasty and stenting (both in combination with medical therapy), or medical therapy alone. Based on a recent systematic review, the optimal treatment strategy for patients with asymptomatic carotid stenosis remains unclear [2].

At the same time, the medical management for asymptomatic carotid stenosis continues to evolve. Contemporary medical therapy primarily includes antiplatelet agents and statins [3-5]. Aspirin is considered the firstline option among antiplatelets for thromboprophylaxis, whereas statins may have beneficial effects on plaque morphology and attenuate the underlying inflammatory response of vulnerable plaques [6]. Furthermore, modifiable vascular risk factors associated with carotid atherosclerosis (e.g., hypertension, diabetes, smoking, physical inactivity, and unhealthy diet) should be screened for and treated according to accepted standards [5].

Medical therapy has evolved over time and the combination of treatments currently considered 'optimal' has not been evaluated as the comparator in available randomized trials of revascularization interventions, because these trials were completed more than 10 years ago [2]. However, the stroke event rates observed with medical therapy alone in older trials are still often used to communicate risks for patients not receiving revascularization and to guide clinical decision-making [7]. Patients are typically presented with an estimated annual risk of ipsilateral carotid territory stroke of $2 \%$ with medical therapy alone, which can be cut in half by endarterectomy, according to the Asymptomatic Carotid Atheroscle- rosis Study (ACAS) trial (published in 1995) [7, 8]. Recent data suggest that contemporary event rates with medical therapy may be much lower $[9,10]$. The American Heart Association/American Stroke Association guidelines on primary stroke prevention state that 'the benefit of surgery may now be lower than anticipated based on randomized trial results' [5]. Thus, accurate estimates of event rates under medical therapy alone are needed to quantify the potential for benefit from invasive procedures and to inform clinical decision-making [11, 12]. Furthermore, risk estimates can be used to inform the design of future comparative studies of alternative treatment options $[9,13]$. If medical treatment results in low event rates, then invasive procedures that pose even a minimal periprocedural risk may be unlikely to yield benefits for many years, despite being highly effective postprocedurally. Thus, trials evaluating the addition of invasive procedures to medical therapy for asymptomatic carotid stenosis will need larger sample sizes and long follow-up periods to attain adequate statistical power [13, 14].

We performed a systematic review of 'best-available evidence' to investigate temporal changes in the incidence rate of cerebrovascular events, mortality, and cardiovascular events among patients with asymptomatic carotid stenosis receiving medical intervention alone $[15$, 16]. We also used estimates of event rates under medical therapy to illustrate their implications for the design of future randomized trials.

\section{Materials and Methods}

This review is based in part on a Health Technology Assessment Report commissioned by the Coverage and Analysis Group at the Centers for Medicare and Medicaid Services (CMS) and prepared by the Tufts Evidence-Based Practice Center under contract with the Agency for Healthcare Research and Quality (AHRQ) [15]. The systematic review component of this work was based on a protocol that predefined the research questions, study selection criteria, and synthesis methods that we used. The full text of the report is available at http://www.ahrq.gov/research/findings/ta/ carotidstenosis/carotidstenosis.pdf (last accessed: July 25, 2013). When applicable, we followed the Meta-Analysis of Observational Studies in Epidemiology (MOOSE) reporting guidelines [17].

\section{Search Strategy}

We searched MEDLINE and the Cochrane Central Register of Controlled Trials (from inception through December 31, 2012) to identify English-language publications reporting the results of cohort studies of patients receiving medical therapy for asymptomatic carotid stenosis. In electronic database searches, we combined terms for carotid stenosis and relevant treatments (medical thera- 
py, endarterectomy, or stenting; the search strategies were designed to address both medical and nonmedical treatments for asymptomatic carotid stenosis because the scope of the Technology Assessment Report, on which this paper is based, included nonmedical therapies). We provide the complete search strategy in online supplementary appendix table 1 (for all online suppl. material, see www.karger.com/doi/10.1159/000365206). We supplemented our searches with a bibliography of single-group cohort studies of medical therapy published before 2008 provided by the author of a previously published systematic review [A.L. Abbott, pers. commun., 9]. In addition, we reviewed the bibliographies of other relevant systematic and narrative reviews and searched the Food and Drug Administration Web site. We contacted the corresponding authors of eligible studies for unpublished data on outcomes of interest if the published information was not sufficient for our analyses.

\section{Study Selection}

A single reviewer screened all abstracts (N.H., G.D.K., D.M., or G.R.) and a second reviewer rescreened abstracts marked as irrelevant. To increase sensitivity, we retrieved the full text of abstracts considered potentially relevant by any reviewer. We selected prospective studies conducted in adults ( $\geq 18$ years) who had asymptomatic carotid artery stenosis (50-99\% atherosclerotic narrowing of the carotid bifurcation lumen or extracranial part of the internal carotid artery) without ipsilateral carotid territory symptoms in the previous 6 months. In general, we accepted the definition of 'asymptomatic stenosis' used in each study. Studies reporting on mixed cohorts of patients (symptomatic and asymptomatic carotid artery stenosis) were included only if results were stratified according to symptom status.

We focused on medical therapy alone, defined broadly to also include lifestyle modification. Specifically, interventions of interest included the following modalities, alone or in combination: use of antiplatelets, anticoagulants, antihypertensives, lipid-lowering drugs, control of diabetes, and lifestyle modification (e.g., smoking cessation, exercise, and dietary changes) [18]. We developed operational definitions of the type and intensity of medical interventions used in each study (e.g., use of dual or single antiplatelet treatment, statins). However, we did not exclude a priori studies that did not report on the medical treatment used or that specifically did not use a certain type of treatment. We also included natural history studies, because we assumed that patients included in such studies with active follow-up did not undergo revascularization interventions and received the 'standard medical care' available at the time. We excluded studies that compared different types of medical treatments and studies that utilized comparisons of interventions with historical controls.

We included studies that reported information on major clinical outcomes: stroke, transient ischemic attack (TIA), death, myocardial infarction, other cardiovascular events, and composite cardiovascular outcomes. We considered ipsilateral carotid territory events (stroke, TIA, or both) to be the outcomes of primary interest, since these events can be directly attributable to the carotid artery under study. We included prospective cohort studies and the medical therapy arm of eligible randomized controlled trials (RCTs) or prospective nonrandomized comparative studies of patients that underwent carotid endarterectomy for ipsilateral asymptomatic or contralateral symptomatic carotid stenosis. If the degree of stenosis was not clearly reported in studies based on ad- ministrative data set analyses, we assumed that all patients had a carotid stenosis of $\geq 50 \%$.

Eligible studies had to have enrolled at least 30 participants with a minimum average follow-up duration of 12 months. Smaller studies and those with shorter follow-up are unlikely to produce results of adequate precision.

A single reviewer (N.H., G.D.K., D.M., or G.R.) examined the full-text publications of potentially relevant studies, and a second reviewer reexamined all studies deemed ineligible (N.H., G.D.K., D.M., G.R., D.E.T., T.F.O., or E.F.). Discrepancies were resolved by consensus involving a third investigator.

\section{Data Extraction}

A single investigator (N.H., G.D.K., D.M., or G.R.) extracted data from each study into electronic forms; a second investigator verified all records. We collected information on study design, funding sources, setting, population characteristics, the definition of carotid stenosis, the imaging modality used and related diagnostic and quality assurance criteria for determining the degree of stenosis, and details regarding medical therapies. For each outcome of interest, we recorded the outcome definitions used, the baseline screening methods, the frequency and duration of follow-up, and the methods for outcome ascertainment.

We calculated incidence rates expressed as events over total person-time (in person-years). If raw data were not reported, data to calculate incidence rates were extracted from published KaplanMeier curves [19] using digitization software (Engauge Digitizer version 2.14; http://digitizer.sourceforge.net/) [20].

\section{Assessment of the Risk of Bias and Completeness of Reporting}

We assessed the risk of bias and completeness of reporting of individual studies on the basis of the following items: study objectives stated clearly; patient selection criteria stated clearly; patients enrolled consecutively; interventions adequately described; outcome definitions provided; rate of dropout or crossover to endarterectomy or stenting of $<20 \%$, and outcome ascertainment by a neurologist $[21,22]$. At least 2 reviewers performed the risk of bias assessment for each study, and discrepancies were resolved by consensus involving a third investigator. We did not combine items into quality scores, because of their well-known limitations [23, 24]. We also extracted information on funding sources.

\section{Statistical Analysis}

Summary (meta-analysis) estimates of the incidence rate of each outcome were obtained by fitting random-effects (randomintercept) generalized linear models, and they were expressed as events per 100 person-years $[25,26]$. We used the exact Poisson likelihood to represent within-study variability. To evaluate temporal changes in the incidence rates of clinical outcomes, we conducted meta-regression analyses by adding 'last year of study recruitment' as a covariate in the model. The effects of other studylevel covariates were evaluated in the same way. Subgroup analyses were conducted based on the severity of carotid artery stenosis, medical treatments received, and last recruitment year.

Statistical analyses were conducted using Stata version IC/12.1 (Stata Corp., College Station, Tex., USA). Statistical significance was defined as a two-sided $\mathrm{p}$ value $<0.05$. We did not perform any adjustments for multiple comparisons [27].
Asymptomatic Carotid Artery Stenosis

Treated with Medical Therapy Alone
Cerebrovasc Dis 2014;38:163-173 DOI: $10.1159 / 000365206$ 
Fig. 1. Search strategy flow diagram. CAS = Carotid artery stenting; CEA = carotid endarterectomy; RCT = randomized controlled trial.

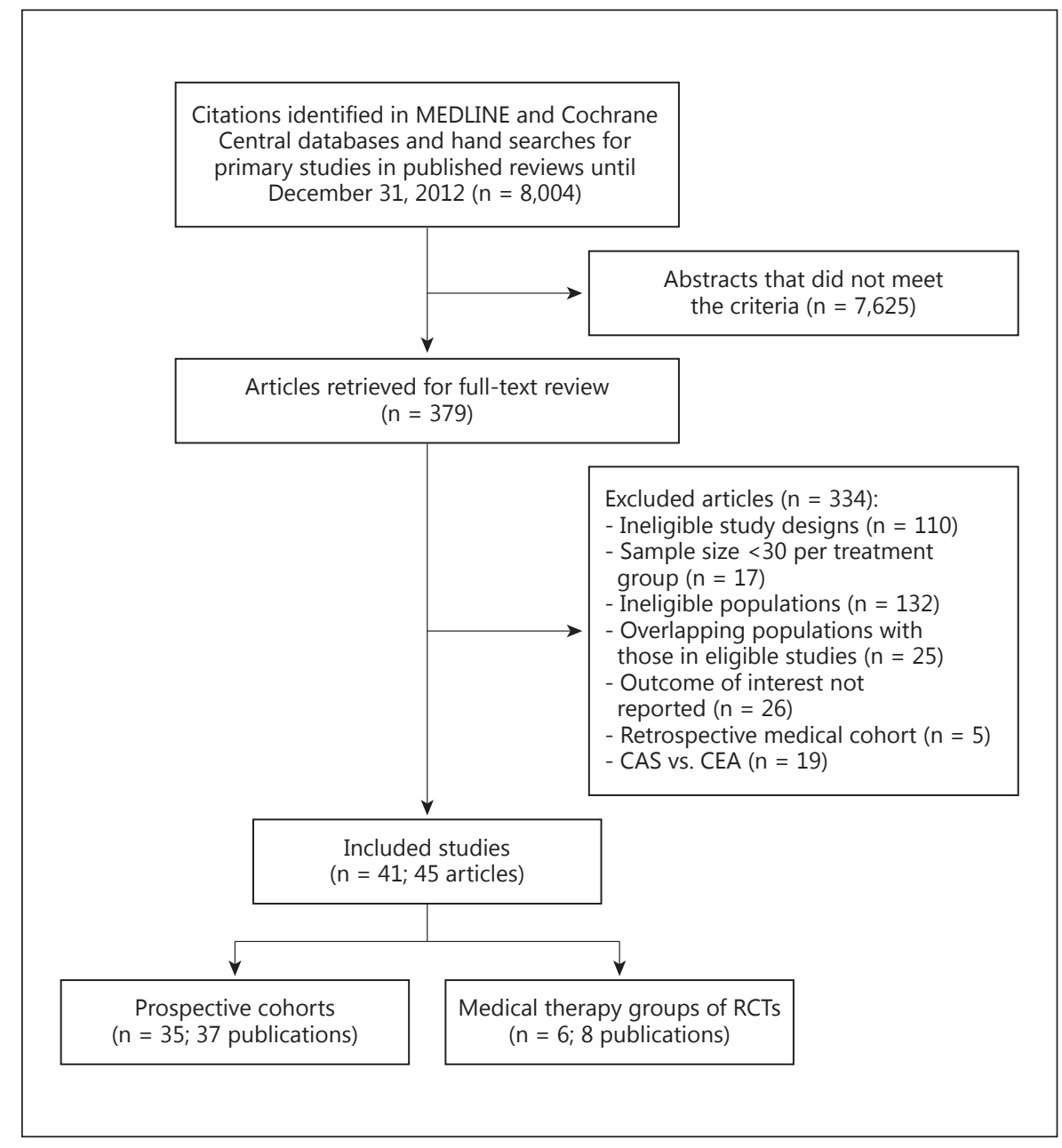

Simulations for the Design of Future Comparative Studies

To explore the implications of our findings for the design of future studies, we performed a series of power calculations under alternative scenarios for the incidence rate in medically treated patients, the incidence rate ratio (IRR; i.e., the effect of the comparator treatment compared to medical therapy), and the sample size of the trials. We simulated parallel-arm trials with incidence rates in the medical treatment group ranging from very low $(0.5$ events per 100 person-years) to very high (10 events per 100 personyears). We assumed 'true' incidence rates in the comparator groups based on a wide range of IRRs (vs. medical therapy), ranging from strong protective effects to no effect (IRR from 0.5 to 1 ). Each trial had a 1:1 randomized design, with 5 years of follow-up and a total sample size ranging from 500 to 5,000 participants. For each scenario (i.e., for each combination of medical therapy event rate, IRR, and sample size), we simulated 1,000 randomized trials and performed a comparison between treatment groups using an exact test for the outcome of 'at least 1 incident event' [28]. Power was calculated as the proportion of simulations where the $p$ value was statistically significant at the $\alpha=0.05$ level [29]. In online supplementary appendix document 1 , we present additional simulation analyses for scenarios where the comparator treatment was as- sumed to have some 'upfront' procedural risk (for the same outcome that the treatment prevented during follow-up), as would be expected for invasive interventions associated with periprocedural adverse events.

\section{Results}

\section{Included Studies}

Our searches identified 8,004 citations, of which 379 were considered potentially relevant and were retrieved in full text. Of these, 45 publications (online suppl. appendix document 2) assessed medical therapy and met our inclusion criteria, contributing 41 independent strata ('studies') to our analyses (fig. 1). Six of the studies (8 publications) were of medically treated groups from RCTs comparing medical therapy and surgical interventions; the remaining 35 studies were prospective cohort studies of medical therapy. 
Table 1. Summary incidence rates by outcome

\begin{tabular}{lcccc}
\hline & Studies, $\mathrm{n}$ & $\begin{array}{l}\text { Summary incidence } \\
\text { rate (95\% CI) }\end{array}$ & $\begin{array}{l}\text { Median number of } \\
\text { events (25th, 75th percentile) }\end{array}$ & $\begin{array}{l}\text { Median person-years of } \\
\text { follow-up (25th, 75th percentile) }\end{array}$ \\
\hline Any stroke & 17 & $2.7(2.0-3.7)$ & $16(7,27)$ & $713.8(386.6,930.0)$ \\
Any stroke or death & 3 & $6.1(4.2-8.9)$ & $43(37,51)$ & $850.4(442.1,936.2)$ \\
Any stroke or TIA & 9 & $5.6(4.0-7.8)$ & $23(16,64)$ & $621.7(223.2,1,106.7)$ \\
Any TIA & 5 & $4.0(3.1-5.1)$ & $7(4,8)$ & $108.5(108.2,175.0)$ \\
Cardiovascular death & 6 & $4.1(3.2-5.2)$ & $40(23,91)$ & $1,069.2(301.0,2,269.2)$ \\
Death & 13 & $4.6(3.4-6.3)$ & $59(15,103)$ & $795.6(488.4,2,251.8)$ \\
Ipsilateral stroke & 25 & $1.7(1.3-2.1)$ & $10(5,15)$ & $490.2(354.1,851.9)$ \\
Ipsilateral stroke or TIA & 19 & $5.5(4.3-6.9)$ & $25(10,51)$ & $401.8(112.1,930.0)$ \\
Ipsilateral TIA & 8 & $2.9(1.9-4.3)$ & $11(6,14.5)$ & $377.9(262.1,447.5)$ \\
Myocardial infarction & 5 & $1.8(0.7-4.6)$ & $14(2,14)$ & $398.0(301.0,538.0)$ \\
\hline
\end{tabular}

Incidence rates are expressed as events per 100 person-years. $\mathrm{CI}=$ Confidence interval.

Eligible studies had completed patient recruitment between 1978 and 2009. Online supplementary appendix table 2 provides details regarding the design and patient population included in each study. The majority of the studies $(\mathrm{n}=22 ; 54 \%)$ did not include any participants who had previously undergone endarterectomy in the contralateral carotid artery; of those, 1 study included patients with contralateral carotid occlusion. Ten other studies (25\%) included at least some patients who had undergone endarterectomy in the contralateral carotid artery (19-100\% of participants). Nine studies (22\%) did not report information regarding prior surgery to the contralateral carotid artery.

The 41 studies had enrolled a total of 16,178 participants followed for an average of 3.4 years (ranging from 1 to 9). The average participant age across all studies was 68 years, and the proportion of males ranged from 40 to $100 \%$, with a median of $63.5 \%$. The prevalence of hypertension, coronary artery disease, and hyperlipidemia ranged from 12 to $90 \%, 14$ to $81 \%$, and 5 to $79 \%$, respectively. The proportion of current or ever-smokers ranged from 14 to $91 \%$.

Online supplementary appendix table 3 summarizes the findings of our assessment of the risk of bias and completeness of reporting for the 41 included studies. Two of the 6 studies derived from medical treatment groups of RCTs were deemed to be at medium risk of bias, and the remaining 4 at low risk of bias. Five, 12, and 18 of the 35 prospective cohort studies were deemed to be at low, medium, and high risk of bias, respectively.

\section{Components of Medical Management}

Twenty-nine of the 41 studies (71\%) reported information on the medical treatment received by their participants; among them, 28 (68\%) reported details of antithrombotic treatment, and 13 (32\%) reported details of lipid-lowering treatment. Among the studies reporting data on the medical therapy received, the median of the proportion of patients using antithrombotic drugs (including both antiplatelet and anticoagulant drugs) was 91.5\% (ranging from 0 to 100). Two studies disallowed participants from taking any antiplatelet drugs. The proportion of patients receiving lipid-lowering medications ranged from 5 to $88 \%$, with a median of $62 \%$. Treatment with statins was more common in recent years: $67 \%$ of the studies completing recruitment from 2000 onwards reported that at least $25 \%$ of their participants received statins, compared to $4 \%$ of the studies ending recruitment earlier ( $p<0.001$ ). Similarly, $73 \%$ of the studies completing recruitment from 2000 onwards reported that at least half of their participants received antithrombotic treatment, compared to $50 \%$ of the studies ending recruitment earlier $(\mathrm{p}=0.14)$.

\section{Meta-Analysis of Incidence Rates and Trends over Time}

Table 1 summarizes the results of meta-analyses of incidence rates for cerebrovascular and cardiovascular outcomes as well as mortality across all studies. Meta-regression analyses (table 2) showed that the incidence rates of ipsilateral stroke and ipsilateral stroke or TIA have declined statistically significantly over time $(p=0.001$ and $\mathrm{p}<0.001$, respectively). In contrast, the last recruitment 
Table 2. Results from the meta-regression analyses

\begin{tabular}{|c|c|c|c|c|}
\hline & ipsilateral stroke & ipsilateral stroke or TIA & any stroke & any stroke or TIA \\
\hline all studies & $0.61(0.45-0.82)$ & $0.60(0.47-0.77)$ & $1.02(0.63-1.64)$ & $0.76(0.43-1.36)$ \\
\hline $\mathrm{p}$ value $\mathrm{a}^{\mathrm{a}}$ & 0.001 & $<0.001$ & 0.956 & 0.353 \\
\hline \multicolumn{5}{|c|}{ Last study recruitment year } \\
\hline $\mathrm{p}$ value ${ }^{\mathrm{b}}$ & $<0.001$ & 0.003 & 0.189 & $<0.001$ \\
\hline \multicolumn{5}{|c|}{ Degree of stenosis } \\
\hline $50-70 \%$ & $1.9(1.5-2.5)(\mathrm{n}=11)$ & $4.9(3.8-6.5)(n=10)$ & $1.9(1.0-3.5)(n=6)$ & $5.6(4.1-7.5)(\mathrm{n}=6)$ \\
\hline$>70 \%$ & $2.1(1.6-2.7)(\mathrm{n}=13)$ & $7.3(5.5-9.6)(\mathrm{n}=12)$ & $4.2(2.1-4.9)(\mathrm{n}=6)$ & $8.92(6.5-12.3)(n=7)$ \\
\hline $\mathrm{p}$ value ${ }^{\mathrm{b}}$ & 0.427 & 0.001 & 0.001 & 0.001 \\
\hline \multicolumn{5}{|c|}{ Proportion of patients receiving antithrombotics } \\
\hline$<50 \%$ & NA $(n=0)$ & $\mathrm{NA}(\mathrm{n}=0)$ & $1.9(0.7-5.3)(n=2)$ & $5.4(2.3-12.7)(\mathrm{n}=2)$ \\
\hline$\geq 50 \%$ & $1.6(1.2-2.3)(\mathrm{n}=16)$ & $4.9(4.0-6.0)(\mathrm{n}=11)$ & $2.9(2.0-4.2)(\mathrm{n}=13)$ & $5.3(3.0-9.4)(\mathrm{n}=4)$ \\
\hline $\mathrm{p}$ value ${ }^{\mathrm{b}}$ & $\mathrm{NA}$ & NA & 0.484 & 0.964 \\
\hline
\end{tabular}

Incidence rates are expressed as events per 100 person-years. Significant differences are given in italics. NA = Not applicable. ${ }^{\mathrm{a}} \mathrm{p}$ values for the effect of 10 years' difference in last recruitment year. ${ }^{\mathrm{b}} \mathrm{p}$ values for the difference between subgroups.

year was not statistically significantly associated with any territory cerebrovascular outcomes. Figure 2 plots the incidence rates of individual studies against the last recruitment year of each study. There was a clear declining trend for ipsilateral stroke and ipsilateral stroke or TIA (39 and $40 \%$ reduction per decade, respectively).

We aimed to explore further why the reduction in incidence rates of ipsilateral events over time was statistically significant, whereas the reductions in rates of any territory events were not. Given that different studies were included in the meta-regression analysis for each outcome (depending on the completeness of reporting of outcome data in each study), we performed sensitivity analyses for the 'any territory' outcomes by considering only those studies that also reported data on ipsilateral outcomes. In these analyses, there was evidence that the incidence rates of any stroke have declined statistically significantly over time ( $\mathrm{p}=0.03$ and 0.05 , respectively).

\section{Subgroup Analyses}

Using meta-regression (table 2), we explored whether studies completing recruitment from 2000 onwards (more representative of contemporary medical therapies) produced different estimates of the rate of ipsilateral cerebrovascular events compared with studies completing recruitment earlier. The summary incidence rate of ipsilateral stroke was significantly decreased in recent studies compared with older studies (1.0 vs. 2.3 per 100 personyears; $\mathrm{p}<0.001)$. Similar results were obtained for the outcome of ipsilateral stroke or TIA ( $\mathrm{p}=0.003)$.

We conducted additional exploratory meta-regression analyses by considering the following covariates (also presented in table 2): the proportion receiving statins ( $\geq 25$ vs. $<25 \%$ ), the proportion receiving antiplatelet treatment ( $\geq 50$ vs. $<50 \%$ ), and the degree of stenosis ( $>70$ vs. $50-70 \%$ stenosis). Studies with a higher proportion of patients using statins resulted in statistically significantly lower incidence rates of ipsilateral events $(\mathrm{p}=0.009)$. Additionally, a higher degree of carotid stenosis ( $>70$ vs. $50-70 \%$ ) was associated with a higher risk of both ipsilateral (stroke or TIA) and any territory outcomes ( $\mathrm{p}$ values for interaction $<0.01$ ). The degree of stenosis was not statistically significantly associated with a higher rate of ipsilateral stroke in the metaregression analyses $(\mathrm{p}=0.43)$. 
Fig. 2. Meta-regression of event rates by last recruitment year for 4 major ischemic cerebrovascular outcomes. Each study is depicted by a hollow circle with a size proportional to the number of observed events (for each outcome). The fitted line is derived from the meta-regression model. a Any stroke. b Any stroke/TIA. c Ipsilateral stroke. d Ipsilateral stroke/TIA.
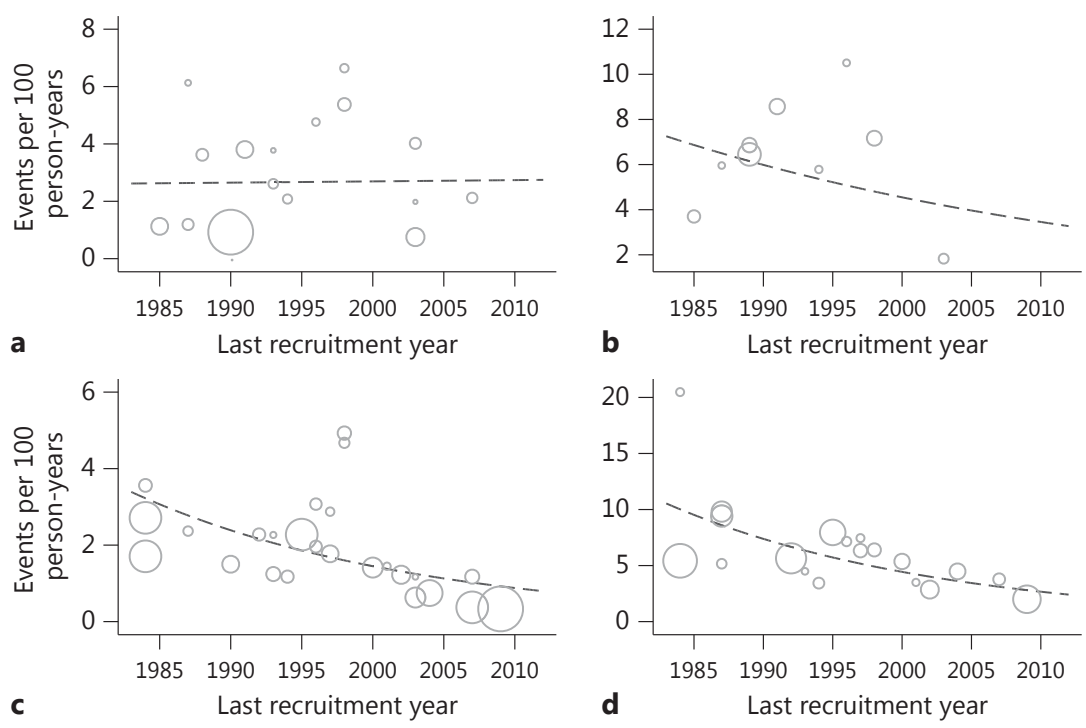

Fig. 3. Results of simulation analyses of study power for different sample sizes and relative treatment effects. Each panel depicts simulations for trials with a different sample size [n $=500$ (a), 1,000 (b), 2,500 (c), or 5,000 (d)]. Within each panel, results are plotted for the following baseline event rates (under medical therapy): $0.5(\times), 1$ $(\diamond), 2(\bigcirc), 3(\boldsymbol{\square}), 5(\triangle)$, and $10(\bullet)$ events per 100 person-years. The $\mathrm{x}$-axis depicts the IRRs comparing the new treatment with medical therapy during the follow-up period.

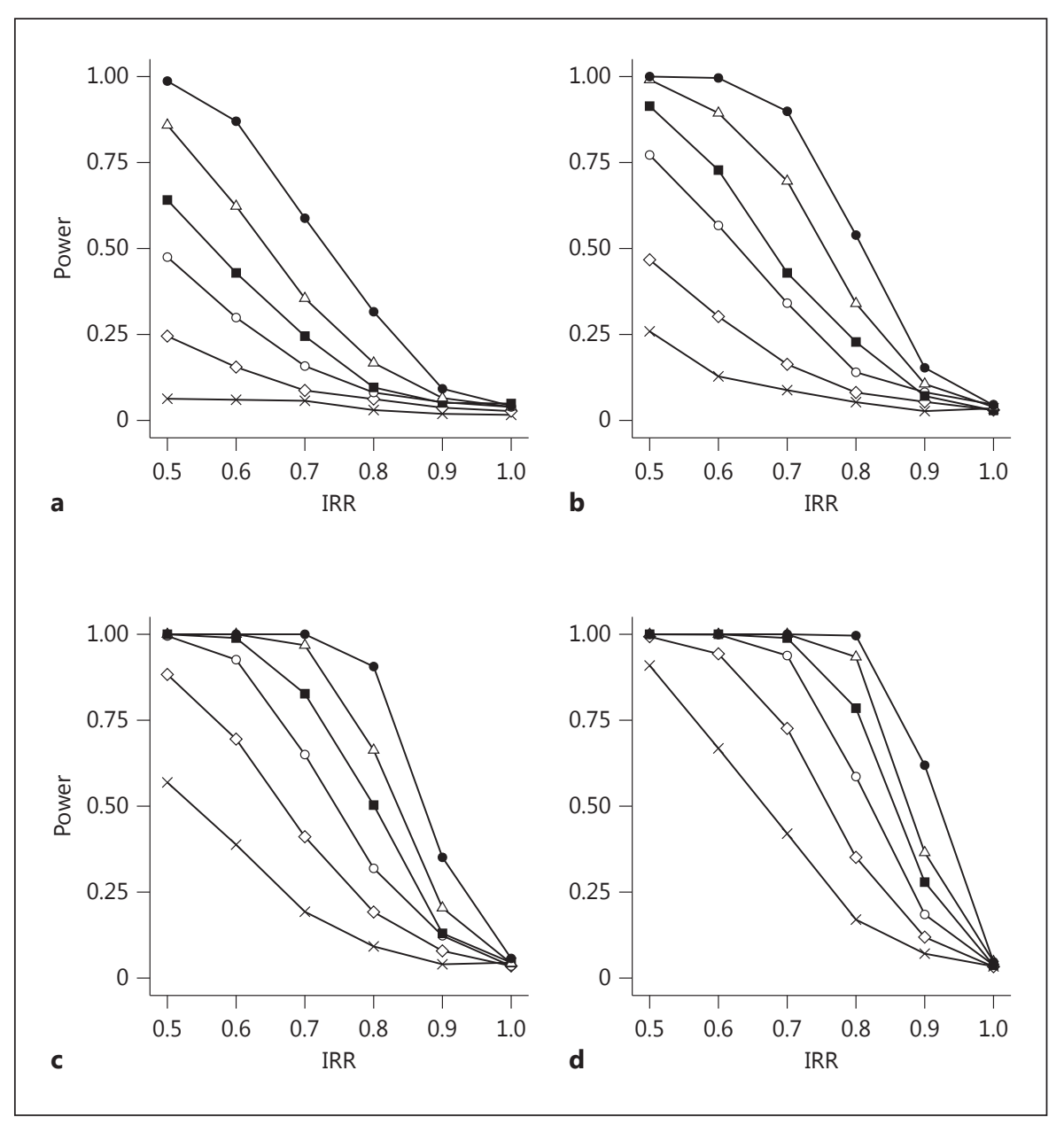

Asymptomatic Carotid Artery Stenosis Treated with Medical Therapy Alone 


\section{Power Analysis for Future Studies}

We explored the implications of our estimates of the incidence rates of different outcomes under medical therapy for the design of future comparative studies using simulations (fig. 3). These analyses indicated that for low-to-medium event rates ( $0.5-1$ event per 100 personyears), studies of up to 5,000 individuals with 5 years of follow-up would be underpowered to detect effect sizes as large as IRR $=0.5$. For higher event rates (e.g., 5-10 events per 100 person-years, as would be expected in trials of selected high-risk patients), studies will have adequate power (e.g., $\geq 80 \%$ ) to reliably detect treatment effects only if sample sizes are fairly large $(\geq 2,500$ patients) and effect sizes are moderate to large (IRR $\leq 0.7$ ). Results were qualitatively similar in simulations where the comparator intervention was assumed to carry some risk of periprocedural adverse events (online suppl. appendix document 1).

\section{Discussion}

Our systematic review indicates that the summary incidence rate of stroke for patients with asymptomatic carotid stenosis treated with medical therapy alone is approximately 1.7 per 100 person-years. In a subgroup analysis, the summary incidence rate of ipsilateral stroke was significantly lower in recent studies (last recruitment year between 2000 and 2010) as compared with studies completing recruitment earlier (1.0 vs. 2.3 events per 100 person-years). Meta-regression analysis indicated that the rates of ipsilateral stroke and ipsilateral stroke or TIA have significantly declined by approximately $40 \%$ per decade over the past 25 years. The majority of older studies included in our analyses had enrolled patients who did not receive treatments consistent with contemporary medical therapy standards (e.g., antithrombotic agents and statins). In the meta-analysis stratified by the reported use of statins, we found that studies in which at least $25 \%$ of the participants were treated with statins had significantly lower rates of ipsilateral stroke than studies in which less than $25 \%$ of the participants were treated with these drugs.

The significant reduction in the rate of ipsilateral events over time is in agreement with previous work [9, 30], including a 2009 systematic review that examined a smaller number of studies $(\mathrm{n}=11)$ using a weighted linear regression model [9]. This review included studies enrolling at least 100 participants, whereas we used a threshold of 30 participants, resulting in the inclusion of a larger number of studies $(n=41)$. Furthermore, we employed meta-analysis models that are more appropriate for the analysis of rare events [25, 26]. Finally, in contrast to the previous review, which examined only trends in the incidence rate over time, our analysis provided summary estimates of absolute event rates that can be used to inform clinical decision-making and the design of future trials.

We found that the last recruitment year was not statistically significantly associated with any territory cerebrovascular outcomes (any stroke and any stroke or TIA). This finding contradicts the previous systematic review, which reported a significant reduction in the published rates of any stroke and any stroke or TIA. The apparent discrepancy may be explained by differences in study selection criteria. The previous review included studies reporting any territory outcomes only if they also provided data on ipsilateral stroke events; in contrast, we included studies with any territory outcome data, irrespective of whether ipsilateral outcomes were reported. When we repeated the analyses for the outcome of any territory stroke limited to studies that also reported information on ipsilateral events, we also found that more proximal last recruitment years were significantly associated with lower incidence rates of any stroke. Discrepancies between analyses using all studies and analyses restricted to studies reporting ipsilateral event rates may be due to differences in outcome ascertainment methods or selective reporting of outcome information.

Our subgroup analysis by degree of stenosis showed that the incidence rate of ipsilateral stroke did not differ statistically significantly between populations with more than $70 \%$ carotid stenosis and those with $50-70 \%$ stenosis, even though the average event rates were somewhat different (2.1 vs. 1.9 per 100 person-years, respectively). The lack of difference may be due to heterogeneity in the definition and measurement of the degree of stenosis across studies [31]. When analyses were stratified by stenosis severity (instead of using meta-regression), the difference became more pronounced (2.4 vs. 1.6 per 100 person-years). Furthermore, the severity of stenosis in medically treated patients was associated with statistically significantly higher summary incidence rates for all other cerebrovascular outcomes (ipsilateral stroke or TIA, any stroke, and any stroke or TIA).

The declining rates of ipsilateral cerebrovascular events over time are a cause for optimism for individuals with asymptomatic carotid stenosis. A similar pattern was reported in a systematic review of RCTs of medical 
therapies for secondary stroke prevention published from 1960 to 2009 [32]. This review showed that over a period of 5 decades, the rates of recurrent stroke and major vascular events declined by 1 and $1.3 \%$ per decade, respectively, in absolute terms. At the same time, declining event rates pose a challenge for the design and conduct of future comparative studies: because most interventions are expected to result in modest relative risk reductions [33], future studies in patients with asymptomatic carotid stenosis will need to enroll large numbers of individuals to attain adequate statistical power in order to detect these modest effect sizes. Alternatively, studies could be conducted in 'enriched' patient populations at high risk of cerebrovascular events with medical therapy alone, since such patients could potentially benefit from invasive revascularization procedures [34]. The identification of such individuals could be based on prognostic risk models that combine patient information with the results of specialized imaging tests [35-38].

Our work has some limitations. First, the populations included in the studies we reviewed were heterogeneous, reflecting variability in the diagnosis and treatment of asymptomatic carotid stenosis. We explored this heterogeneity in meta-regression analyses and used randomeffects models to account for any residual unexplained variability. Second, the use of study level results, rather than individual patient data, precluded a detailed assessment of the impact of risk factors (e.g., smoking, diabetes status, and family history of cerebrovascular disease) on event rates. Nonetheless, using studies as the unit of analysis was appropriate for our primary aim of assessing the evolution of event rates in medically treated populations over time. Third, the studies reported limited information regarding the details of the medical treatments administered to enrolled patients, limiting our ability to directly evaluate longitudinal treatment patterns and their impact on prognosis. Changes in study level event rates may be explained by multiple causes, beyond changes in treatment patterns. For example, changes in the diagnostic approach to asymptomatic carotid stenosis [31, 39, $40]$ or changes in the distribution of risk factors in the population [33, 41-43] could account for our findings. Finally, our simulation analyses did not account for participant withdrawals or other causes of censoring. In general, in the presence of noninformative censoring, the power would be even lower; thus, our results represent a best-case (optimistic) scenario for the design of future studies.

\section{Conclusions}

Our analyses show that the prognosis of patients with asymptomatic carotid stenosis receiving medical treatment alone has improved over time, with the rates of ipsilateral cerebrovascular events almost halving during the period covered by our review. It appears that changes in diagnosis, the availability of effective medical therapies, or risk factor modifications have resulted in substantial declines in the rates of vascular events among patients with asymptomatic carotid stenosis. At the same time, this success has rendered the conduct of future research more challenging because trials will have to be much larger in order to reliably detect small or modest treatment effects of novel treatments.

\section{Acknowledgements}

We thank Dr. A.L. Abbott (School of Public Health and Preventive Medicine, The Alfred Centre, Monash University, Melbourne, Vic., Australia) for providing the list of included and excluded studies from her earlier review [9]. We thank Drs. D.M. Kent (Tufts Medical Center, Boston, Mass., USA) and T.A. Trikalinos (Brown University, Providence, R.I., USA) for providing helpful comments on previous versions of the manuscript.

This paper is based on a Health Technology Assessment Report prepared by the Tufts Evidence-Based Practice Center under contract with the AHRQ, Rockville, Md., USA (contract No. HHSA 290200710055 I). The findings and conclusions in this paper are those of the authors, who are responsible for its content, and do not necessarily represent the views of the AHRQ or CMS. No statement in this report should be construed as an official position of the AHRQ, the US Department of Health and Human Services, or the CMS.

\section{Disclosure Statement}

The authors have no conflicts of interest to declare.

\begin{tabular}{|c|c|}
\hline References & $\begin{array}{l}\text { Rijbroek A, Wisselink W, Vriens EM, Barkhof } \\
\text { F, Lammertsma AA, Rauwerda JA: Asymp- } \\
\text { tomatic carotid artery stenosis: past, present } \\
\text { and future. How to improve patient selection? } \\
\text { Eur Neurol 2006;56:139-154. } \\
\text { 2 Raman G, Moorthy D, Hadar N, Dahabreh IJ, } \\
\text { O'Donnell TF, Thaler DE, Feldmann E, Lau J, } \\
\text { Kitsios GD: Management strategies for as- } \\
\text { ymptomatic carotid stenosis: a systematic re- } \\
\text { view and meta-analysis. Ann Intern Med } \\
\text { 2013;158:676-685. }\end{array}$ \\
\hline
\end{tabular}


-3 Brott TG, Halperin JL, Abbara S, Bacharach JM, Barr JD, Bush RL, Cates CU, Creager MA, Fowler SB, Friday G, Hertzberg VS, McIff EB, Moore WS, Panagos PD, Riles TS, Rosenwasser RH, Taylor AJ: 2011 ASA/ACCF/AHA/ AANN/AANS/ACR/ASNR/CNS/SAIP/ SCAI/SIR/SNIS/SVM/SVS guideline on the management of patients with extracranial carotid and vertebral artery disease: executive summary. A report of the American College of Cardiology Foundation/American Heart Association Task Force on Practice Guidelines, and the American Stroke Association, American Association of Neuroscience Nurses, American Association of Neurological Surgeons, American College of Radiology, American Society of Neuroradiology, Congress of Neurological Surgeons, Society of Atherosclerosis Imaging and Prevention, Society for Cardiovascular Angiography and Interventions, Society of Interventional Radiology, Society of Neurointerventional Surgery, Society for Vascular Medicine, and Society for Vascular Surgery. Circulation 2011;124:489532.

4 Brott TG, Halperin JL, Abbara S, Bacharach JM, Barr JD, Bush RL, Cates CU, Creager MA, Fowler SB, Friday G, Hertzberg VS, McIff EB, Moore WS, Panagos PD, Riles TS, Rosenwasser RH, Taylor AJ: 2011 ASA/ACCF/AHA/ AANN/AANS/ACR/ASNR/CNS/SAIP/ SCAI/SIR/SNIS/SVM/SVS guideline on the management of patients with extracranial carotid and vertebral artery disease. A report of the American College of Cardiology Foundation/American Heart Association Task Force on Practice Guidelines, and the American Stroke Association, American Association of Neuroscience Nurses, American Association of Neurological Surgeons, American College of Radiology, American Society of Neuroradiology, Congress of Neurological Surgeons, Society of Atherosclerosis Imaging and Prevention, Society for Cardiovascular Angiography and Interventions, Society of Interventional Radiology, Society of Neurointerventional Surgery, Society for Vascular Medicine, and Society for Vascular Surgery. Circulation 2011;124:e54-e130.

-5 Goldstein LB, Bushnell CD, Adams RJ, Appel LJ, Braun LT, Chaturvedi S, Creager MA, Culebras A, Eckel RH, Hart RG, Hinchey JA, Howard VJ, Jauch EC, Levine SR, Meschia JF, Moore WS, Nixon JV, Pearson TA: Guidelines for the primary prevention of stroke: a guideline for healthcare professionals from the American Heart Association/American Stroke Association. Stroke 2011;42:517-584.

-6 Alsheikh-Ali AA, Kitsios GD, Balk EM, Lau J, Ip S: The vulnerable atherosclerotic plaque: scope of the literature. Ann Intern Med 2010; 153:387-395.
7 Silver B, Zaman IF, Ashraf K, Majed Y, Norwood EM, Schuh LA, Smith BJ, Smith RE, Schultz LR: A randomized trial of decisionmaking in asymptomatic carotid stenosis. Neurology 2012;78:315-321.

-8 Endarterectomy for asymptomatic carotid artery stenosis. Executive Committee for the Asymptomatic Carotid Atherosclerosis Study. JAMA 1995;273:1421-1428.

$\checkmark 9$ Abbott AL: Medical (nonsurgical) intervention alone is now best for prevention of stroke associated with asymptomatic severe carotid stenosis: results of a systematic review and analysis. Stroke 2009;40:e573-e583.

10 Spence JD: Asymptomatic carotid stenosis. Circulation 2013;127:739-742.

11 White CJ, Jaff MR: Catch-22: carotid stenting is safe and effective (Food and Drug Administration) but is it reasonable and necessary (Centers for Medicare and Medicaid Services)? JACC Cardiovasc Interv 2012;5:694-696.

12 Abbott AL, Adelman MA, Alexandrov AV, et al: Why calls for more routine carotid stenting are currently inappropriate: an international, multispecialty, expert review and position statement. Stroke 2013;44:1186-1190.

13 Kent DM, Trikalinos TA: Therapeutic innovations, diminishing returns, and control rate preservation. JAMA 2009;302:2254-2256.

14 Scannell JW, Blanckley A, Boldon H, Warrington $\mathrm{B}$ : Diagnosing the decline in pharmaceutical R\&D efficiency. Nat Rev Drug Discov 2012;11:191-200.

15 Raman G, Kitsios GD, Moorthy D, Hadar N, Dahabreh IJ, O'Donnell TF, Thaler DE, Feldmann E, Lau J: Management of asymptomatic carotid stenosis. Bethesda, Agency for Healthcare Research and Quality, 2012.

$>16$ Paulus JK, Dahabreh IJ, Balk EM, Avendano EE, Lau J, Ip S: Opportunities and challenges in using studies without a control group in comparative effectiveness reviews. Res Synth Methods 2014;5:152-161.

17 Stroup DF, Berlin JA, Morton SC, Olkin I, Williamson GD, Rennie D, Moher D, Becker BJ, Sipe TA, Thacker SB: Meta-analysis of observational studies in epidemiology: a proposal for reporting. Meta-Analysis of Observational Studies in Epidemiology (MOOSE) group. JAMA 2000;283:2008-2012.

18 Wadden TA, Webb VL, Moran CH, Bailer BA: Lifestyle modification for obesity: new developments in diet, physical activity, and behavior therapy. Circulation 2012;125: $1157-1170$

19 Parmar MK, Torri V, Stewart L: Extracting summary statistics to perform meta-analyses of the published literature for survival endpoints. Stat Med 1998;17:2815-2834.

20 Shadish WR, Brasil IC, Illingworth DA, White KD, Galindo R, Nagler ED, Rindskopf DM: Using UnGraph to extract data from image files: verification of reliability and validity. Behav Res Methods 2009;41:177-183.
21 Ip S, Paulus JK, Balk EM, Dahabreh IJ, Avendano EE, Lau J: Role of single group studies in Agency for Healthcare Research and Quality comparative effectiveness reviews, ed 2013/02/22. Report No 13-EHC007-EF. Rockville, Agency for Healthcare Research and Quality, 2013.

22 Kitsios GD, Dahabreh IJ, Abu Dabrh AM, Thaler DE, Kent DM: Patent foramen ovale closure and medical treatments for secondary stroke prevention: a systematic review of observational and randomized evidence. Stroke 2012;43:422-431.

23 Greenland S: Invited commentary: a critical look at some popular meta-analytic methods. Am J Epidemiol 1994;140:290-296.

24 Greenland S, O’Rourke K: On the bias produced by quality scores in meta-analysis, and a hierarchical view of proposed solutions. Biostatistics 2001;2:463-471.

25 Hamza TH, van Houwelingen HC, Stijnen T: The binomial distribution of meta-analysis was preferred to model within-study variability. J Clin Epidemiol 2008;61:41-51.

26 Stijnen T, Hamza TH, Ozdemir P: Random effects meta-analysis of event outcome in the framework of the generalized linear mixed model with applications in sparse data. Stat Med 2010;29:3046-3067.

27 Rothman KJ: No adjustments are needed for multiple comparisons. Epidemiology 1990; 1: 43-46.

28 Fisher RA: The logic of inductive inference. J R Stat Soc 1935;98:39-82.

29 Feiveson A: Power by simulation. Stata J 2002; 2:107-124

30 Naylor AR: What is the current status of invasive treatment of extracranial carotid artery disease? Stroke 2011;42:2080-2085.

-31 Toole JF, Castaldo JE: Accurate measurement of carotid stenosis. Chaos in methodology. J Neuroimaging 1994;4:222-230.

- 32 Hong KS, Yegiaian S, Lee M, Lee J, Saver JL: Declining stroke and vascular event recurrence rates in secondary prevention trials over the past 50 years and consequences for current trial design. Circulation 2011;123:21112119.

33 Yusuf S, Collins R, Peto R: Why do we need some large, simple randomized trials? Stat Med 1984;3:409-422.

34 Bogiatzi C, Cocker MS, Beanlands R, Spence JD: Identifying high-risk asymptomatic carotid stenosis. Expert Opin Med Diagn 2012; 6:139-151.

35 Kakkos SK, Griffin MB, Nicolaides AN, Kyriacou E, Sabetai MM, Tegos T, Makris GC, Thomas DJ, Geroulakos G: The size of juxtaluminal hypoechoic area in ultrasound images of asymptomatic carotid plaques predicts the occurrence of stroke. J Vasc Surg 2013;57: 609-618.e1, discussion 617-618. 
36 Momjian-Mayor I, Kuzmanovic I, Momjian S, Bonvin C, Albanese S, Bichsel D, Comelli M, Pereira VM, Lovblad KO, Sztajzel RF: Accuracy of a novel risk index combining degree of stenosis of the carotid artery and plaque surface echogenicity. Stroke 2012;43:12601265.

37 Topakian R, King A, Kwon SU, Schaafsma A, Shipley M, Markus HS: Ultrasonic plaque echolucency and emboli signals predict stroke in asymptomatic carotid stenosis. Neurology 2011;77:751-758.

38 Madani A, Beletsky V, Tamayo A, Munoz C, Spence JD: High-risk asymptomatic carotid stenosis: ulceration on 3D ultrasound vs TCD microemboli. Neurology 2011;77:744-750.
39 Feinstein AR, Sosin DM, Wells CK: The Will Rogers phenomenon. Stage migration and new diagnostic techniques as a source of misleading statistics for survival in cancer. N Engl J Med 1985;312:1604-1608.

40 Sytkowski PA, Kannel WB, D’Agostino RB: Changes in risk factors and the decline in mortality from cardiovascular disease. The Framingham Heart Study. N Engl J Med 1990; 322:1635-1641.

41 Rothwell PM, Coull AJ, Giles MF, Howard SC, Silver LE, Bull LM, Gutnikov SA, Edwards P, Mant D, Sackley CM, Farmer A, Sandercock PA, Dennis MS, Warlow CP, Bamford JM, Anslow P: Change in stroke incidence, mortality, case-fatality, severity, and risk factors in Oxfordshire, UK from 1981 to 2004 (Oxford Vascular Study). Lancet 2004;363: 1925-1933.
2 Tolonen H, Mahonen M, Asplund K, Rastenyte D, Kuulasmaa K, Vanuzzo D, Tuomilehto J: Do trends in population levels of blood pressure and other cardiovascular risk factors explain trends in stroke event rates? Comparisons of 15 populations in 9 countries within the WHO MONICA Stroke Project. World Health Organization Monitoring of Trends and Determinants in Cardiovascular Disease. Stroke 2002;33:2367-2375.

43 Harmsen P, Wilhelmsen L, Jacobsson A: Stroke incidence and mortality rates 1987 2006 related to secular trends of cardiovascular risk factors in Gothenburg, Sweden. Stroke 2009;40:2691-2697. 